\title{
O cuidado da família à pessoa renal crônica em diálise peritoneal
}

\author{
Care of the family to the chronic kidney \\ person in peritoneal dialysis
}

\begin{abstract}
Daniella Marques Negreiros' • Angelina Monteiro Furtado ${ }^{2}$ - Cristiano Batista Gonçalves ${ }^{3}$ Ítalo Arão Pereira Ribeiro ${ }^{4}$ Laianny Luize Lima e Silva ${ }^{5}$. Anderson de Assis Ferreira ${ }^{6}$ Andréa da Silva Araújo ${ }^{7}$ • João Paulo da Silva Batista ${ }^{8}$ 'Ranielly Alencar Barbosa ${ }^{9}$
\end{abstract}

\section{RESUMO}

Objetivou-se analisar as evidências científicas sobre o papel da família no cuidado a pessoa com insuficiência renal crônica em diálise peritoneal. Estudo do tipo revisão integrativa, o qual foi utilizado as seguintes bases de dados: Banco de Dados em Enfermagem (BDENF) e Literatura Latino Americana e do Caribe em Ciências da Saúde (LILACS), ambas via Biblioteca Virtual em Saúde (BVS); e biblioteca virtual: Scientific Eletronic Library Online - SciELO. Para busca dos estudos utilizou da estratégia da descrição adaptada de PVO. Para essa pesquisa foram utilizados os seguintes descritores controlados: "Insuficiência Renal Crônica", "Diálise Peritoneal", "Tratamento domiciliar", "Família","Resultado do tratamento", "Eficácia". Os artigos estudados foram agrupados em 3 categorias: na realização da diálise em si, no apoio emocional e social na colaboração financeira. A participação da família é marcada pelo envolvimento ativo no tratamento, pela responsabilização da qualidade de vida, adesão ao tratamento e incentivadora do esquema terapêutico.

Palavras-chave: Diálise Peritoneal; Família; Enfermagem.

\section{ABSTRACT}

The objective was to analyze the scientific evidence on the role of the family in the care of the person with chronic renal failure in peritoneal dialysis. An integrative review study, which used the following databases: Nursing Database (BDENF) and Latin American and Caribbean Literature in Health Sciences (LILACS), both via Virtual Health Library (VHL); andVirtual Library: Scientific Eletronic Library Online - SciELO. For studies search used the strategy of adapted PVO description. For this research, the following controlled descriptors were used: "Chronic Renal Insufficiency", "Peritoneal Dialysis", "Home Treatment", "Family", "Treatment Result", "Efficacy." The articles were grouped into 3 categories: the participation of the family is marked by the active involvement in the treatment, by the accountability of the quality of life, adherence to the treatment and incentive of the therapeutic scheme.

Keywords: Peritoneal Dialysis; Family; Nursing. 


\section{INTRODUÇÃO}

A insuficiência renal crônica (IRC) é uma patologia que afeta os diferentes aspectos da vida do paciente. É de difícil tratamento, com sérias implicações físicas, psicológicas e socioeconômicas, não apenas para o indivíduo, mas como para a família e sociedade (I). Quando o indivíduo é diagnosticado com IRC faz-se necessário a utilização de algum tratamento que substitua a função dos rins. Dentre os tratamentos disponíveis estão: hemodiálise, diálise peritoneal (DP) e transplante renal. Apesar de a escolha ser do paciente ou responsável legal, muitas vezes não é possível ofertar todas as opções( ${ }^{(2)}$.

A DP consiste na infusão, permanência e drenagem de solução na cavidade peritoneal, por meio de um cateter flexível implantado no abdome. Este processo promove a retirada de excesso de líquido corpóreo e de substâncias tóxicas, que seriam normalmente eliminadas por meio da urina. A DP é realizada em hospitais especializados ou no domicílio dos pacientes. Nesta terapia são realizadas quatro trocas diárias ${ }^{(3)}$.

Nessa modalidade é necessário avaliar não só o paciente, mas também seus familiares para identificar se estão aptos a realizar esse tratamento, visto que o cliente e a família serão os responsáveis diretos, uma vez que o mesmo poderá ser realizado no domicilio. É realizada uma capacitação, que consiste em aulas teóricas e práticas, com objetivo de qualificar o paciente e seus familiares para realizarem o procedimento técnico no domicílio com segurança( ${ }^{(4)}$.

Quando se inicia a diálise peritoneal, todo processo pelo qual o paciente será submetido mudará os padrões de vida de toda família diante da nova realidade ${ }^{(5)}$. Fazendo-se necessário incluir os membros familiares dentro do processo de cuidado e no plano assistencial de enfermagem, os quais muitas vezes acabam sendo esquecidos.

Nesse sentido, a enfermagem não pode desconsiderar a problemática vivenciada pelas famílias que tem um familiar em DP. Muitas situações difíceis enfrentadas pela família, talvez sejam consequência de uma assistência de enfermagem que pouco corresponda às suas reais necessidades, uma vez que o enfoque ainda permanece essencialmente no indivíduo doente e nos aspectos técnicos para realização da $D P^{(5)}$.

Considerando tais aspectos, essa pesquisa apresenta como objetivo analisar as evidências científicas sobre a participação da família no cuidado à pessoa com insuficiência renal crônica em diálise peritoneal.

\section{MÉTODO}

Trata-se de uma revisão integrativa, guiada por seis etapas de investigação: definição do tema e elaboração da questão de pesquisa; amostragem, busca na literatura e delimitação para a inclusão dos estudos; extração de dados; avaliação crítica dos estudos incluídos; análise e síntese dos resultados; e apresentação da revisão ou síntese do conhecimento( ${ }^{(7)}$.

$\mathrm{Na}$ elaboração da questão clinica utilizou-se a estratégia PVO, composta de 3 elementos básicos na confecção da indagação, contemplando a presença de fatores fundamentais, assegurando, assim, que todos os aspectos da incerteza clínica estejam incluídos na questão formulada. Tais elementos são: População (população, contexto e/ou situação-problema), Variáveis (quando houver) e Resultado - Outcomes resultado (desejado ou indesejável); conhecidos pela sigla PVO(8), resultando na seguinte pergunta: quais as evidências científicas sobre a participação da família no cuidado à pessoa com insuficiência renal crônica em diálise peritoneal?
Para busca das produções científicas, foram utilizados os seguintes vocábulos controlados disponíveis nos Descritores em Ciências da Saúde (DeCS): "Insuficiência Renal Crônica", "Diálise Peritoneal", "Tratamento domiciliar", "Família", "Resultado do tratamento", "Eficácia”, "Avaliação de EficáciaEfetividade de Intervenções". Para esses descritores fora realizado o cruzamento utilizando os conectores booleanos AND e OR. Conforme a estratégia ilustrada no Quadro 2.

Quadro I - Descritores utilizados na estratégia PVO para busca de artigos sobre o papel da família da pessoa que realiza diálise peritoneal. Floriano, PI, Brasil, 2017.

\begin{tabular}{|c|c|}
\hline ELEMENTOS PVO & DESCRITORES DECS \\
\hline $\begin{array}{c}\text { Pessoa com IRC em diálise } \\
\text { peritoneal } \\
\text { AND }\end{array}$ & $\begin{array}{c}\text { Insuficiência renal crônica OR Diálise } \\
\text { peritoneal OR Tratamento domiciliar }\end{array}$ \\
\hline $\begin{array}{c}\text { V-Variável } \\
\text { Presença da família } \\
\text { AND }\end{array}$ & Família \\
\hline $\begin{array}{c}\text { O - Resultado } \\
\text { efeito da presença da } \\
\text { família no tratamento }\end{array}$ & $\begin{array}{c}\text { Resultado do tratamento OR Eficácia } \\
\text { OR Avaliação de Eficácia-Efetividade de } \\
\text { Intervenções }\end{array}$ \\
\hline
\end{tabular}

O levantamento da literatura foi realizado entre os meses de janeiro a março de 2017, nas seguintes bases de dados: Banco de Dados em Enfermagem (BDENF) e Literatura Latino Americana e do Caribe em Ciências da Saúde (LILACS), ambas via Biblioteca Virtual em Saúde (BVS); e biblioteca virtual: Scientific Eletronic Library Online - SciELO.

Foram incluídos estudos que se encontravam disponíveis on-line, sem restrição de idiomas e que respondessem a questão de pesquisa. Sendo excluídos os estudos duplicados, sendo considerados apenas uma vez. Não houve definição de recorte temporal, na tentativa de resgatar o maior número de publicações disponíveis sobre a temática.

Os artigos foram acessados por meio do portal de periódicos da Coordenação de Aperfeiçoamento de Pessoal de Nível Superior (CAPES) e a busca, seleção e inclusão realizada por dois revisores de forma independente que procederam com a leitura de títulos e resumos de modo a garantir maior rigor metodológico e fidedignidade dos resultados.

Foram recuperadas 42 produções, dentre as quais 30 atenderam aos critérios de inclusão, sendo selecionadas para o estudo. Destaca-se que, 19 artigos foram excluídos por duplicidade nas bases de dados, resultando na amostra de II publicações. A Figura I descreve o percurso realizado para identificação, inclusão e exclusão dos estudos, segundo base consultada.

Figura I - Percurso para identificação, inclusão e exclusão nas bases eletrônicas investigadas.

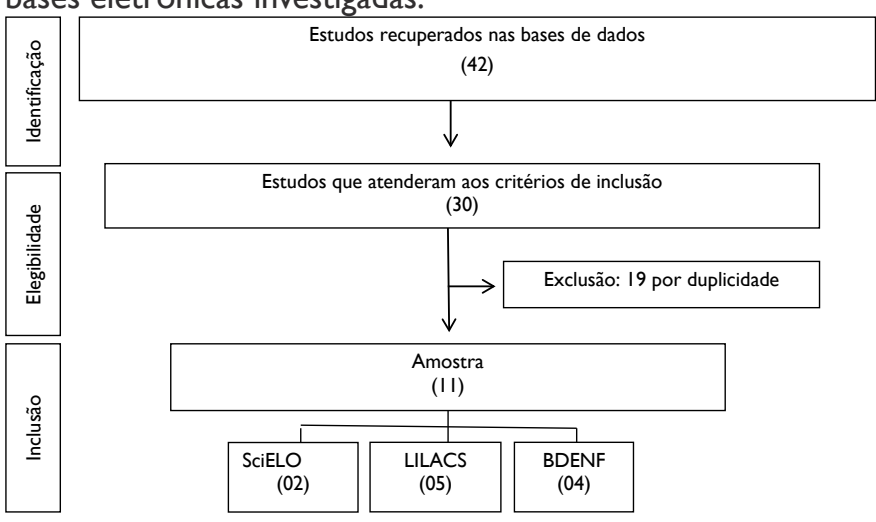


A coleta dos dados foi realizada com auxílio de um instrumento próprio que contemplou variáveis relacionadas à identificação dos estudos (autores e ano de publicação), aspectos metodológicos (delineamento e amostra), atuação da família, efeito da atuação da família e nível de evidência.

Os dados foram classificados de acordo com os níveis de evidências de acordo com os níveis de evidências citados pelos autores ${ }^{(9)}$, a saber:nível I - evidências resultantes da meta-análise de múltiplos estudos clínicos controlados e randomizados; 2 evidências obtidas em estudos individuais com delineamento experimental; 3 - evidências de estudos quase-experimentais; 4 - evidências de estudos descritivos (não-experimentais) ou com abordagem qualitativa; 5 - evidências provenientes de relatos de caso ou de experiência; e 6 - evidências baseadas em opiniões de especialistas.

A análise crítica e a síntese dos resultados foram realizadas de forma descritiva, o que possibilitou a classificação dos estudos por similaridade semântica e a construção de três grupos temáticas.

\section{RESULTADOS}

Dentre as II produções incluídas, todas tiveram suas publicações variando entre os anos de 2003 a 2016 . Houve maior prevalência de publicações durante o ano de 2010 (3 artigos). Dessa forma, verificou-se que os pesquisadores incluídos nesse estudo começaram a publicar só nas últimas duas décadas, evidenciando uma produção relativamente recente dessa temática.

Ao fazer a análise dos níveis de evidência, constatou-se que os estudos são classificados com o nível 4 ( 9 artigos), nível 5 ( I artigo) e nível 6 ( I artigo), demonstrando a escassez e a necessidade da realização de maiores pesquisas com rigor metodológico mais forte. Entre a amostra estudada variou de 7 a I5I participantes por estudo, fazendo parte desses grupos adultos, crianças e adolescentes.

Tais achados podem ser observados no Quadro 3, apresentando a descrição dos estudos incluídos nessa revisão por Autor/ano de publicação, nível de evidência (NE), delineamento de pesquisa e amostra.

Quadro 2- Caracterização das publicações quanto o nível de evidência, metodologia e tipo de estudo. Floriano, PI, Brasil, 2017.

\begin{tabular}{|c|c|c|c|}
\hline Autor/ ano & NE & $\begin{array}{c}\text { Delineamento } \\
\text { de pesquisa }\end{array}$ & Amostra \\
\hline Timm et al., 2015 & 4 & $\begin{array}{c}\text { Qualitativo } \\
\text { descritivo } \\
\text { e exploratório }\end{array}$ & $\begin{array}{l}15 \text { pessoas } \\
\text { (todos adultos). }\end{array}$ \\
\hline $\begin{array}{l}\text { Paula; Nascimento; } \\
\text { Rocha, } 2008 .\end{array}$ & 4 & Qualitativo & $\begin{array}{c}14 \text { participantes } \\
\text { (crianças e adultos) }\end{array}$ \\
\hline $\begin{array}{l}\text { Paula; Nascimento; } \\
\text { Rocha, } 2009\end{array}$ & 5 & Estudo de caso & $\begin{array}{c}4 \text { famílias, } \\
\text { totalizando } \\
14 \text { participantes. }\end{array}$ \\
\hline $\begin{array}{l}\text { Tavares; Lisboa, } \\
2015 .\end{array}$ & 4 & $\begin{array}{l}\text { Descritivo, com } \\
\text { abordagem } \\
\text { qualitativa. }\end{array}$ & 9 participantes. \\
\hline Tavares et al., 2016 & 4 & $\begin{array}{l}\text { Descritivo, com } \\
\text { abordagem } \\
\text { qualitativa. }\end{array}$ & 19 familiares. \\
\hline $\begin{array}{l}\text { Abrahão et al., } \\
2010\end{array}$ & 4 & $\begin{array}{l}\text { Descritivo com } \\
\text { abordagem } \\
\text { quantitativa. }\end{array}$ & $\begin{array}{l}30 \text { pacientes, } \\
\text { crianças } \\
\text { e adolescentes. }\end{array}$ \\
\hline
\end{tabular}

\begin{tabular}{|c|c|c|c|}
\hline Abrahão, 20I0. & 4 & $\begin{array}{c}\text { Descritivo com } \\
\text { abordagem } \\
\text { quantitativa. }\end{array}$ & $\begin{array}{c}30 \text { crianças } \\
\text { e adolescentes. }\end{array}$ \\
\hline Simpionato, 2005. & 4 & Descritivo & $\begin{array}{c}13 \text { membros } \\
\text { familiares }\end{array}$ \\
\hline $\begin{array}{c}\text { Villarreal-rios, } \\
2014 .\end{array}$ & 4 & $\begin{array}{c}\text { Descritivo com } \\
\text { abordagem } \\
\text { quantitativa. }\end{array}$ & 151 pessoas. \\
\hline $\begin{array}{c}\text { Carreira; Marcon } \\
2003\end{array}$ & 4 & $\begin{array}{c}\text { Descritivo- } \\
\text { exploratório, } \\
\text { método } \\
\text { qualiquantitativo }\end{array}$ & 16 famílias. \\
\hline Lomba, et al.,2014 & 6 & $\begin{array}{c}\text { Revisão da } \\
\text { literatura }\end{array}$ & 7 artigos \\
\hline
\end{tabular}

Fonte: Elaborado pelos pesquisadores.

A maior parte das citações sobre o papel da família na diálise, encontrada nos estudos inclusos nesta pesquisa, destaca o quão é importante esse papel na vida das pessoas que realizam DP como tratamento para mantê-los vivos. Sobre esse papel destacam-se as seguintes categorias: a realização da DP em si, o apoio emocional e social e colaboração financeira. É possível observar isso no Quadro 4.

Quadro 4- Categorização da produção científica quanto à atuação da família da pessoa com IRC em DP. Floriano, PI, Brasil, 2017.

\begin{tabular}{|c|c|c|}
\hline AUTOR/ANO & $\begin{array}{l}\text { ATUAÇÃO } \\
\text { DA FAMÍLIA }\end{array}$ & $\begin{array}{l}\text { EFEITO DA ATUAÇÃO DA } \\
\text { FAMILIA }\end{array}$ \\
\hline $\begin{array}{l}\text { Timm et al., 2015; Paula; } \\
\text { Nascimento; Rocha, } \\
\text { 2008; tavares et al., 2016/ } \\
\text { abrahão et al., 2010/ } \\
\text { abrahão, 2010/ simpionato, } \\
2005 .\end{array}$ & $\begin{array}{l}\text { Na realização da } \\
\text { DP em si }\end{array}$ & $\begin{array}{l}\text { Realização correta da } \\
\text { técnica utilizada na diálise. } \\
\text { Responsabilização pela adesão ao } \\
\text { tratamento, principalmente nos } \\
\text { casos de crianças com IRC. Sucesso } \\
\text { obtido no tratamento mesmo com } \\
\text { as dificuldades iniciais. Garantia } \\
\text { de um plano integral e efetivo } \\
\text { elaborado com a participação da } \\
\text { família. Por outro lado, adesão } \\
\text { inapropriada ao tratamento devido } \\
\text { a insatisfação dos familiares por } \\
\text { serem os únicos responsáveis pelo } \\
\text { tratamento; técnica inadequada } \\
\text { feitas pelas mães devido o cansaço } \\
\text { e falta de atenção. }\end{array}$ \\
\hline $\begin{array}{l}\text { Timm et al., 20I5; Paula; } \\
\text { Nascimento; Rocha, 2008; } \\
\text { Paula; Nascimento; Rocha, } \\
\text { 2009; Tavares; Lisboa, } \\
\text { 20I5; Tavares et al., 2016; } \\
\text { Carreira; Marcon, 2003; } \\
\text { Lomba, et al., 2014 }\end{array}$ & $\begin{array}{c}\text { Apoio emocional } \\
\text { e social }\end{array}$ & $\begin{array}{c}\text { Aumento do vínculo e } \\
\text { comunicação familiar. Bem-estar } \\
\text { psicológico da pessoa doente. } \\
\text { Conformação com a nova condição } \\
\text { imposta. Melhora a espiritualidade } \\
\text { e as crenças religiosas. Motivação } \\
\text { para seguir firme no tratamento. } \\
\text { Sem apoio familiar, o paciente } \\
\text { apresenta tristeza, depressão } \\
\text { e sentimento de abandono. A } \\
\text { dependência excessiva contribui } \\
\text { para não adequação do tratamento. }\end{array}$ \\
\hline $\begin{array}{c}\text { Timm et al., 20I5; Paula; } \\
\text { Nascimento, Rocha, 2008; } \\
\text { Villarreal-Rios, 20I4; } \\
\text { Abrahão, 20 I0; Carreira; } \\
\text { Marcon, } 2003\end{array}$ & $\begin{array}{l}\text { Colaboração } \\
\text { financeira }\end{array}$ & $\begin{array}{l}\text { Manter financeiramente a pessoa } \\
\text { no tratamento. Diminuí as } \\
\text { preocupações geradas por não ter } \\
\text { condição para arcar com despesas } \\
\text { extras. Quando não há uma } \\
\text { articulação para que ocorra essa } \\
\text { colaboração, isso gera desconforto } \\
\text { entre os membros familiares, } \\
\text { interferindo no negativamente no } \\
\text { tratamento. }\end{array}$ \\
\hline
\end{tabular}

\section{DISCUSSÃO}

A preocupação dos membros da família com a capacitação para realizar a DP de forma segura e eficiente no domicilio é organizada definindo o papel de cada membro, identificando aqueles que serão responsáveis pela $\mathrm{DP}^{(5)}$. Quando se trata 
especificamente de famílias de crianças que realizam DP, as mães mantêm todos seus esforços focados no tratamento de seus filhos com objetivo de mantê-los vivos ${ }^{(10)}$, já outra pesquisa mostrou que apesar de todas as dificuldades encontradas no início do tratamento foram superadas com auxilio próprio ou com auxílio da equipe de enfermagem, possibilitando um resultado benéfico no tratamento do seu familiar(II).

Com o tempo, os familiares de clientes em DP conseguem lidar com as adversidades impostas pela doença, e a aceitação do tratamento reflete na disposição em auxiliar e cuidar do seu parente no domicílio(12). Para a realização da DP, a pesquisa dos autores $^{(13)}$ identificou que a mãe era a responsável exclusiva em $24(80 \%)$ casos sendo que apenas 3 (10\%) das mães podiam contar com o pai e/ou tia e em I (1/3) desses casos, com a própria paciente; em 2 (7\%) casos o pai foi o responsável pela DP, em I (3\%) a avó paterna do paciente.

Com as novas rotinas as famílias vivenciam em suas vidas o consumo de tempo e com relação a isso, as repercussões decorrentes das exigências da diálise peritoneal levam as famílias se sentirem aprisionadas e viverem em liberdade condicionada(5). Estudo avaliou a qualidade da aplicação da técnica de DP, realizada por cuidadores em crianças e adolescentes assistidos, revelando a inadequação em $60 \%$ $\operatorname{casos}^{(14)}$. Pesquisadores ${ }^{(6)}$ verificaram que excesso de tarefas a serem desempenhadas, principalmente pelas mães, faz com que o cuidador tenha um grande cansaço físico e, até, psíquico, que pode originar falha no processo diálise e dano para o paciente.

Outra parte dos incômodos é associada à dificuldade na realização da técnica correta do tratamento, que se trata de algo nunca visto pelas famílias e podem gerar medo. Mas isto poderia ser sanado com o apoio da enfermagem na capacitação da família, esclarecendo as dúvidas e receios que possam surgir.

Estudos mostram o quão à família é importante nas relações sociais e no apoio emocional da pessoa com IRC que realiza DP.Após o adoecimento de um membro, os familiares se uniram mais, aumentaram a comunicação e tiveram seus vínculos fortalecidos, contribuindo para apoio emocional e afetivo não só doente como os demais(5). Contar com o apoio de todos os familiares foi uma maneira que as mães e os pais de crianças com IRC encontraram para se tranquilizarem e se sentirem confortáveis ${ }^{(15)}$. Pesquisa realizada pelos autores ${ }^{(13)}$, revelou que, apesar de toda adversidade enfrentada, os familiares procuram manter-se unidos no propósito de cultivar relações familiares saudáveis apesar de qualquer dificuldade.

Quando não se pode contar com um membro ou ajuda familiar para solucionar algum problema, isso gera um sentimento de desamparo e potencializam as dificuldades para pessoa doente, além disso, a dependência do familiar doente pode gerar desconforto tanto para família quanto para - membro familiar envolvido no cuidado(6). Nesse sentido, não receber ajuda ou apoio da família no tratamento, pode ocasionar sentimento de abandono, revolta e depressão, sentido por pessoas que fazem dialise peritoneal e sentem-se sem motivação para viver(16).

Estudo feito por Carreira e Marcon ${ }^{(17)}$, buscou saber a importância do trabalho para os familiares e para os membros doentes em DP, sendo constatadas que $37,5 \%$ dos entrevistados experimentam sentimentos de dependência de outras pessoas e do tratamento traduzidas pela falta de liberdade. $O$ cansaço do cuidador da pessoa em DP pode estar relacionado com a dependência do membro familiar doente, também sofre por esta nessa condição, causando sobrecarga emocional e psicossocial, além de queixas pelo isolamento dos amigos tanto para cuidador, como também para o doente (18).

São as famílias que dão suporte nas questões financeiras visando contemplar o orçamento e eventuais despesas como medicações, hospitalizações, alimentação e entre outros. Como muitas vezes os membros da família nuclear ficam sem trabalhar para poder cuidar do filho doente, eles ressaltaram a importância da família estendida na importância do apoio financeiro6. Pesquisa realizada no México identificou que o custo anual da DP para a família foi em média de US\$2.38I,04. Esse gasto refere à medicação em sua maior parte, mas também estão inclusos gastos com alimentação, transporte, internação( ${ }^{(19)}$.

O fator financeiro tinha relação direta com a ausência das atividades laborais por parte dos familiares cuidadores ${ }^{(13)}$. $O$ afastamento das atividades laborais e domésticas em função do adoecimento e da necessidade de realizar a diálise peritoneal no domicílio pode repercutir em sentimento de culpa no familiar doente ${ }^{(6)}$.

Quando a pessoa começa o tratamento dialítico na maioria das vezes ela deixa de trabalhar devido o tratamento, mesmo tendo direito a benefício social para ter condição de tratamento, muitas vezes esse benefício não é suficiente, a colaboração financeira por parte da família é de grande importância no tratamento. Além disso, podem ocorrer casos em que as famílias têm que diminuir as horas trabalhadas ou deixar o emprego para se dedicar a cuidar desse membro da família com DRC. Esse impacto financeiro pode refletir na moradia, alimentação, lazer entre outras coisas que depende de dinheiro(5).

\section{CONCLUSÃO}

Os artigos estudados nessa pesquisa mostraram o papel da família da pessoa com insuficiência renal crônica na DP. Contudo, a participação da família assume papeis como participação ativa no tratamento, de apoio emocional e social, contribuição financeira, responsabilização assim pela qualidade de vida e adesão ao tratamento, incentivadora do esquema terapêutico, estimuladora de auxílio e apoio ao doente renal.

Este estudo apresenta limitações devido à subjetividade do tema e pouca quantidade de estudos encontrados sobre esta temática e a classificação quanto os níveis de evidências, assim, considera-se importante estimular investigações acerca do papel da família da pessoa com insuficiência renal crônica na DP.

Ademais, importa salientar, que este estudo poderá contribuir com novas pesquisas acerca do tema, influenciando para elaboração de maiores investigações e da atuação da enfermagem perante $o$ doente renal e sua família, agregando novos saberes e conhecimentos na oferta de um assistencialismo mais qualificado, holístico e humanizado.

\section{REFERÊNCIAS}

1. Lata AGB, Albuquerque JG, Carvalho LASBP, Lira ALBC. Diagnósticos de enfermagem em adultos em tratamento de hemodiálise. Acta paul. enferm. [Internet]. 2008 [acesso em: 10 de Jul. de 2019]; 2l(esp): 160-163. Disponível em: http://www. scielo.br/scielo.php?script=sci_arttext\&pid=SOI0321002008000500004\&lng=en. http://dx.doi. org/I0.1590/S0103-21002008000500004.

2. Riella MC. Princípios de nefrologia e distúrbios 
hidroeletrolíticos. 5.ed. Rio de Janeiro: Guanabara Koogan; 2010.

3. Daugirdas JT, Blake PG, Ing, TS. Manual de Diálise. Tradução de Telma Lúcia de Azevedo Hennemann. 4.ed. Rio de Janeiro: Guanabara Koogan, 2010.

4. Barros E. et al. Nefrologia: rotinas, diagnóstico e tratamento. 3.ed. Porto Alegre:Artmed, 2006.

5. Amaral TB. As Consequências Sociais relacionadas ao aumento de pacientes com Doença Renal Crônica em terapia Renal substitutiva. Caderno Saúde e Desenvolvimento. [Internet]. 2016 [acesso em: 10 de jul. de 2019]; 9 (5): 85-95. Disponível em: https:// www.uninter.com/cadernosuninter/index.php/saudee-desenvolvimento/article/view/445

6. Timm AMB, Beuter M, Girardon-Perlini NMO, Pauletto MR, Santos Nodos, Bruinsma JL. Liberdade condicionada: repercussões na família ao conviver com um membro em diálise peritoneal. Rev Rene. [Internet]. 2015 [acesso em: I0 de Jul. de 20 I9]; I6 (4): 540-548. Disponível em: http://www.periodicos.ufc.br/ rene/article/view/2746

7. Whittemore R, Knafl K.The integrative review: Updated methodology. J Adv Nurs. [Internet]. 2005 [acesso em: I0 de Jul. de 2019]; 52(5): 546-53. Disponível em: https://doi.org/ I0. I I I I/j. I 365-2648.2005.0362 I.x

8. Buriel EP. Transformando a pesquisa em estratégia de busca. I Oficina de pscicologia baseada em evidencias da USP. [Internet]. 2015 [acesso em: I 2 de Jul. de 20I7]. Disponível em: www.ip.usp.br Pereira AL, Bachion MM. Atualidades em revisão sistemática de literatura, critérios de força e grau de recomendação de evidência. Revista Gaúcha de Enfermagem. [Internet]. 2006 [acesso em: I0 de Jul. de 2019]; 27(4): 49I. Disponível em: https://seer.ufrgs.br/RevistaGauchadeEnfermagem/ article/view/4633

9. Paula ES, Nascimento LC, Rocha SMM. A influência do apoio social para o fortalecimento de famílias com crianças com insuficiência renal crônica. Rev Latino-am Enfermagem [Internet]. 2008 [acesso em: 10 de Jul. de 2019]; I6(4). Disponível em: http://www. scielo.br/scielo.php?script=sci_arttext\&pid=S0 $104-$ I | 692008000400007\&lng=en. http://dx.doi. org/ I0.I590/SO I04- I I692008000400007.

10. Tavares JMAB, Lisboa MTL, Ferreira MdeA, Valadares GV, Silva FVCe. Diálise peritoneal: cuidado familiar ao cliente renal crônico em tratamento no domicílio. Rev Bras Enferm [Internet]. 2016 [acessado em: II de Jul. de 2019]; 69(6): I I 72-I I 78.Available from: http://www. scielo.br/scielo.php?script=sci_arttext\&pid=S00347|67201600060| I 72\&lng=en. http://dx.doi. org/l 0.1590/0034-7| 67-2016-0262.

11. Lisboa MTL, Branco JM.A.A família e o cliente em diálise peritoneal: repercussões do cuidado no domicílio. Seminário Nacional De Pesquisa De Enfermagem. [Internet]. 2013 [acesso em: 18 de mai. De 2017]. Disponível em: http://www.abeneventos.com.br

12. Abrahão SS, Ricas J, Andrade DF, Pompeu FC, Chamahum L,Araújo TM et al. Dificuldades vivenciadas pela família e pela criança/adolescente com doença renal crônica. J. Bras. Nefrol. [Internet]. 2010 [acesso em: I3 Jul. de 2019]; 32(I): I8-22. Disponível em: http://www.scielo.br/scielo.php?script=sci_
arttext\&pid=SOI $01-28002010000100004 \& \operatorname{lng}=e n$. http://dx.doi.org/I0.1590/S0I0I-28002010000I00004.

13. Abrahão SS, Ricas J, Andrade DF, Pompeu FC, Chamahum L, Araújo TM et al. Estudo descritivo sobre a prática da diálise peritoneal em domicílio. J. Bras. Nefrol. [Internet]. 2010 [acesso em: I3 Jul de 2019]; 32(I): 45-50. Disponível em: http://www. scielo.br/scielo.php?script=sci_arttext\&pid=SOI $0 \mathrm{I}$ 28002010000100009\&lng=en. http://dx.doi. org/I0.1590/S0I0I-28002010000100009.

14. Paula ÉSde, Nascimento LC, Rocha SMM. Religião e espiritualidade: experiência de famílias de crianças com Insuficiência Renal Crônica. Rev. bras. enferm. [Internet]. 2009 [acesso em: I3 Jul. de 2019]; 62(I): 100-106. Available from: http://www. scielo.br/scielo.php?script=sci_arttext\&pid=S0034$7 \mid 672009000100015 \&$ Ing=en. org/I0.1590/S0034-7I6720090001000I5.

15. Tavares JMAB, Lisboa MTL. Tratamento com diálise peritoneal: a prática do autocuidado no contexto familiar. Rev enferm UERJ. [Internet]. 2015 [acesso em: 13 de Jul. de 2019]; 23(3): 344-349. Disponível em: https://www.e-publicacoes.uerj.br/index.php/ enfermagemuerj/article/view/5 I 32

16. Carreira L, Marcon SS. Cotidiano e trabalho: concepções de indivíduos portadores de insuficiência renal crônica e seus familiares. Rev. Latino-Am. Enfermagem [Internet]. 2003 [acesso em: I3 de Jul. de 2019]; I I(6): 823-83I. Disponível em: http://www. scielo.br/scielo.php?script=sci_arttext\&pid=SO $104-$ I 16920030006000 I8\&Ing=en. http://dx.doi. org/I0.1590/SOI04-I I6920030006000 I8.

17. Silva HG, Silva MJ da. Motivações do paciente renal para a escolha a diálise peritoneal ambulatorial contínua. REE [Internet]. 2006 [citado I3 de Jul. de 2019]; 5(I). Disponível em: https://www.revistas.ufg.br/fen/article/ view/774

18. Villarreal-Ríos E, Cárdenas-Maldonado C, VargasDaza ER, Galicia-Rodríguez L, Martínez-González L, Baca-Baca R. Institutional and familial cost of patients in continuous ambulatory peritoneal dialysis. Rev. Assoc. Med. Bras. [Internet]. 2014 [citado I3 de Jul. de 2019]; 60(4): 335-34I. Disponível em: http://www. scielo.br/scielo.php?script=sci_arttext\&pid=SO 104 423020 I 4000400335\&lng=en. http://dx.doi. org/I0.1590/I806-9282.60.04.012. 